\title{
QKD terminal for Canada's Quantum Encryption and Science Satellite (QEYSSat)
}

H. Podmore, I. D'Souza, J. Cain, T. Jennewein, B. Higgins, et al.

H. Podmore, I. D'Souza, J. Cain, T. Jennewein, B. L. Higgins, Y. S. Lee, A. Koujelev, D. Hudson, A. McColgan, "QKD terminal for Canada's Quantum Encryption and Science Satellite (QEYSSat)," Proc. SPIE 11852, International Conference on Space Optics - ICSO 2020, 118520H (11 June 2021); doi: $10.1117 / 12.2599162$

SPIE Event: International Conference on Space Optics - ICSO 2021, 2021, Online Only 


\section{International Conference on Space Optics-ICSO 2020}

Virtual Conference

30 March-2 April 2021

Edited by Bruno Cugny, Zoran Sodnik, and Nikos Karafolas
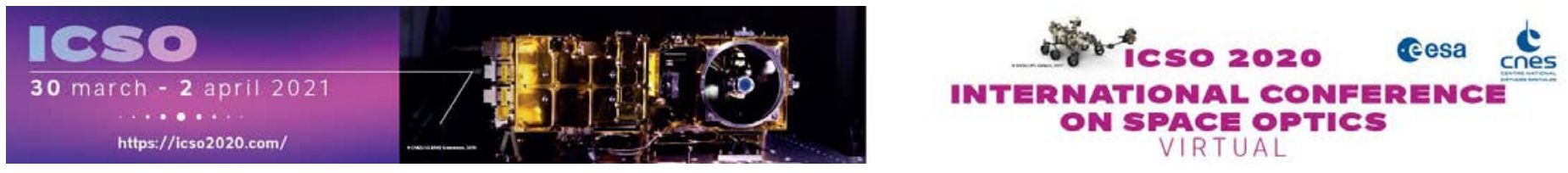

\section{QKD terminal for Canada's Quantum Encryption and Science Satellite (QEYSSat)}

\section{Cesa isopueatans lecnes}




\title{
QKD terminal for Canada's Quantum Encryption and Science Satellite (QEYSSat)
}

\author{
H. Podmore*a, I. D’Souza ${ }^{\text {a }}$, J. Cain ${ }^{\mathrm{a}}$, T. Jennewein ${ }^{\mathrm{b}}$, B. L. Higgins ${ }^{\mathrm{b}}$, Y. S. Lee ${ }^{\mathrm{b}}$, A. Koujelev ${ }^{\mathrm{c}}$, D. \\ Hudson $^{\mathrm{a}}$, A. McColgan ${ }^{\mathrm{a}}$ \\ ${ }^{a}$ Honeywell Aerospace, 303 Terry Fox Dr, Ottawa, ON, Canada, K2K 3J1 \\ binstitute for Quantum Computing, Department of Physics and Astronomy, University of Waterloo, \\ 200 University Ave W, Waterloo, ON, Canada, N2L 3G1 \\ 'Canadian Space Agency, 6767 Route de 1'Aeroport, Saint-Hubert, QC, Canada, J3Y 8Y9
}

\begin{abstract}
Honeywell Aerospace is implementing on behalf of the Canadian Space Agency the Quantum Encryption and Science Satellite (QEYSSat), a Canadian-owned and operated scientific and technology demonstration mission aimed at developing the next-generation of secure communications infrastructure backed by quantum physics. The mission management is led by the Canadian Space Agency and the science is led by the Institute for Quantum Computing at the University of Waterloo. Quantum key distribution (QKD) is a method for issuing, via single-photon transmission, verifiably-confidential encryption keys between two parties. This capability is a powerful tool for the transfer of sensitive data (e.g. financial transactions, health records, etc.), however current terrestrial QKD networks are limited to a few hundred kilometres in geographic reach between nodes. The QEYSSat mission will use a satellite receiver as a trusted node to demonstrate the distribution of secure keys between ground stations separated by at least $400 \mathrm{~km}$. In addition, Honeywell intends to fly an optical intersatellite link (OISL) terminal as a hosted payload on this mission.

The QEYSSat mission will utilize both weak coherent pulse (WCP) sources and entangled photon sources in an uplink configuration to study the performance of QKD, and to perform Bell tests of long-range quantum entanglement. Honeywell is building the QKD receiver terminal consisting of a front-end telescope, a precision pointing and tracking system and single-photon detectors. Major technical challenges include polarization management throughout the optical chain, accurate pointing and tracking, and suppression of background and stray light sources.
\end{abstract}

To address these challenges, Honeywell is leveraging its existing commercial optical communications solutions to meet the more stringent performance requirements for space-based QKD. The QKD terminal architecture consists of an afocal front-end telescope, a wide FOV high-precision pointing and tracking assembly, a polarization analyzer and single-photon photodetector system. A large-diameter on-axis telescope for geostationary optical communications forms the basis for the terminal's front-end optics, and Honeywell's commercial Optical Pointing and Tracking Relay Assembly for intersatellite Communications (OPTRAC) has been adapted as a quantum-ready pointing and tracking unit (QTRAC). For each element, substantial effort has been made to develop an optical system that preserves single-photon states with high fidelity despite the large number of optical surfaces in the chain.

The optical assembly for the QKD terminal was developed and tested at the breadboard level in 2020; this paper will highlight the development and testing of these units as well as the overall architecture and concept of the QEYSSat mission.

Keywords: Quantum Key Distribution, Quantum Networks, Free-space optical communications, LEO constellations

\section{MISSION OVERVIEW}

The QEYSSat mission is a Canadian experiment in the applications of quantum information theory towards secure communications as well as a verification of the boundary range of the theory itself. The two primary activities of the QEYSSat mission are (1) demonstrate a cryptanalytically unbreakable method of encryption-key exchange (employing quantum mechanical principles) that can be used for global-scale secure communications networks, and (2) test the nonlocality of the theory of quantum mechanics itself [1]. To achieve these objectives, the QEYSSat mission will use polarized photons to create a series of quantum links between multiple ground stations and a satellite in low Earth orbit (LEO). These links will then be used to establish shared, secure encryption keys between the ground station users and will also be used 
for performing long-distance Bell tests. These objectives have been refined over the years through multiple concept studies and demonstrations of principle [2] [3].

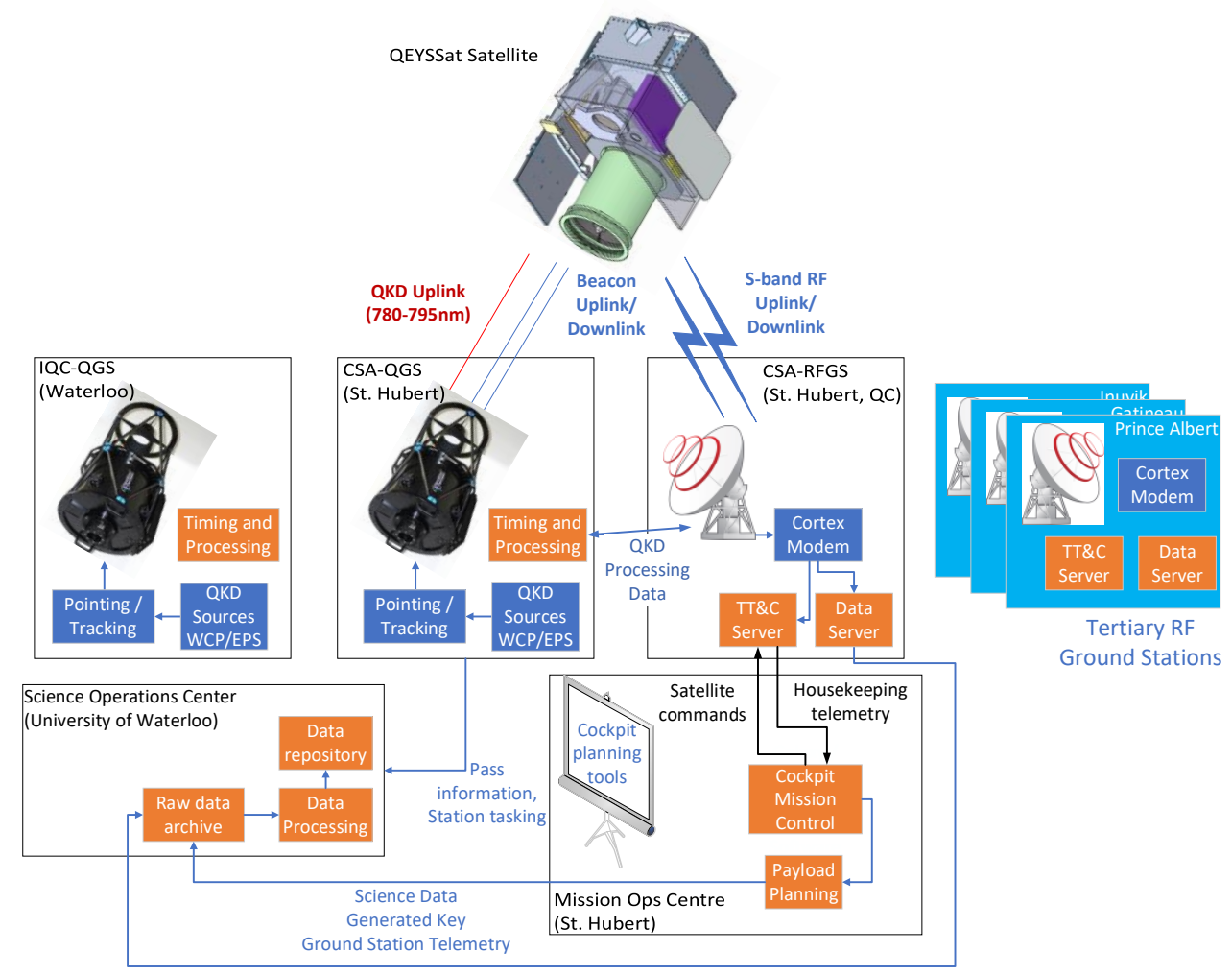

Figure 1. QEYSSat mission architecture. The satellite hosts the QKD terminal and acts as a trusted node in order to produce a shared encryption key between two ground stations separated by over $600 \mathrm{~km}$ (e.g. The University of Waterloo, and The Canadian Space Agency).

The QKD terminals on the satellite and the ground-station will follow the well-established BB84 and BBM92 protocols to establish a secret encryption key between the satellite and user A (a ground station or a remote location connected to a ground station through another quantum channel). Then, a second secure key will be established between the satellite and user B located at a second ground station physically distanced from ground station A by over $400 \mathrm{~km}$. A secure key between user A and user B, will then be prepared on the satellite by calculating a Boolean combination of the two individual keys, and the result is transmitted (over a classical data channel) to one of the users. This user can then calculate the other user's key using the combined key received over the classical channel, and the knowledge of their own key which was communicated securely. For example, user B would receive the combined A and B keys, and reverse the Boolean operation using key B, which they already knew, to recover key A which is now a key that is known to both users.

Unlike previous space demonstrations [4], the QKD terminal on QEYSSat is designed primarily as a receiver for quantum uplinks from ground to the satellite. This architecture minimizes the complexity of the satellite, as locating the quantum source at the ground station shifts the heavy processing burden to the ground station and allows for flexibility in the choice of uplink quantum sources (provided that polarization states are used as the basis for information exchange). Flexibility in source selection is important at the mission level as it allows for testing novel, non-space-approved sources, and supports future upgrades (e.g. quantum repeater-based communication). Additionally, flexibility in source selection has been prioritized over flexibility in receiver selection since detector technologies are relatively mature, while new quantum sources are frequently being developed. While the QKD terminal is configured primarily as a receiver, an additional QKD source on board the terminal is also planned to demonstrate downlink capability. During the first scientific phase of the mission, weak coherent pulse (WCP) and entangled photon uplink sources will be tested with the satellite terminal using ground stations in Canada. This phase of the mission will be used to address the mission objectives of demonstrating QKD over large distances, as well as testing the limits of non-locality via Bell tests. Once the first phase of the mission is completed, the QEYSSat team will expand the base of collaborating users to encompass international partners. 
Both quantum key distribution (QKD) and the Bell tests are well understood and have frequently been performed in a terrestrial setting. The critical elements of the mission design for QEYSSat therefore apply to the specific difficulty in completing the single-photon-exchanges to and from a LEO QKD terminal moving at an angular rate of approximately 1 $\mathrm{deg} / \mathrm{s}$ (as seen from the ground station), at an orbital altitude up to $600 \mathrm{~km}$.

The principal challenge involved in the single-photon exchange arises from the difficulty of detecting an extremely weak signal from a space platform. Since there is no multiplicity of transmitted photon states, individual photons, sent at particular times, must be received over $600 \mathrm{~km}$ away and detected above the inherent background noise of the channel and detectors. Thus, the QKD terminal for the spacecraft must be capable of exacting spatial, spectral, and temporal filtering of the received signal. In other words, the QKD terminal must be designed to provide precise and accurate time-tagging and polarizationanalysis of received photons, narrow spectral filtering about the QKD transmission band, precise pointing of the receiver, and accurate knowledge of the location, velocity and distance between the spacecraft and ground station. In this paper, the provisions for each of these capabilities in the context of the overall QKD terminal will be presented and discussed.

\section{QKD TERMINAL}

The QEYSSat QKD terminal architecture is shown in Figure 2. The terminal comprises a telescope, pointing and tracking assembly, quantum receiver, quantum and beacon sources, and support electronics. The downlink beacon sources provide a tracking target for the ground terminal, and similarly an uplink beacon from the ground station is received at the telescope, and split off within the Quantum Tracking Relay Assembly for Communication (QTRAC) towards a tracking sensor where it provides the signal for the payload acquisition and tracking system. The quantum uplink signal is captured by the telescope, stabilized by the fine-tracking system and passed to the quantum receiver consisting of a polarization analyzer fiber-coupled to a five-channel single-photon detector assembly (four active channels, one reference), and high-speed time tagging and data recorder. The quantum source generates a weak coherent pulse for downlink which is passed through the QTRAC and out of the telescope towards the ground. This payload architecture has been validated in previous studies including airborne trials [3].
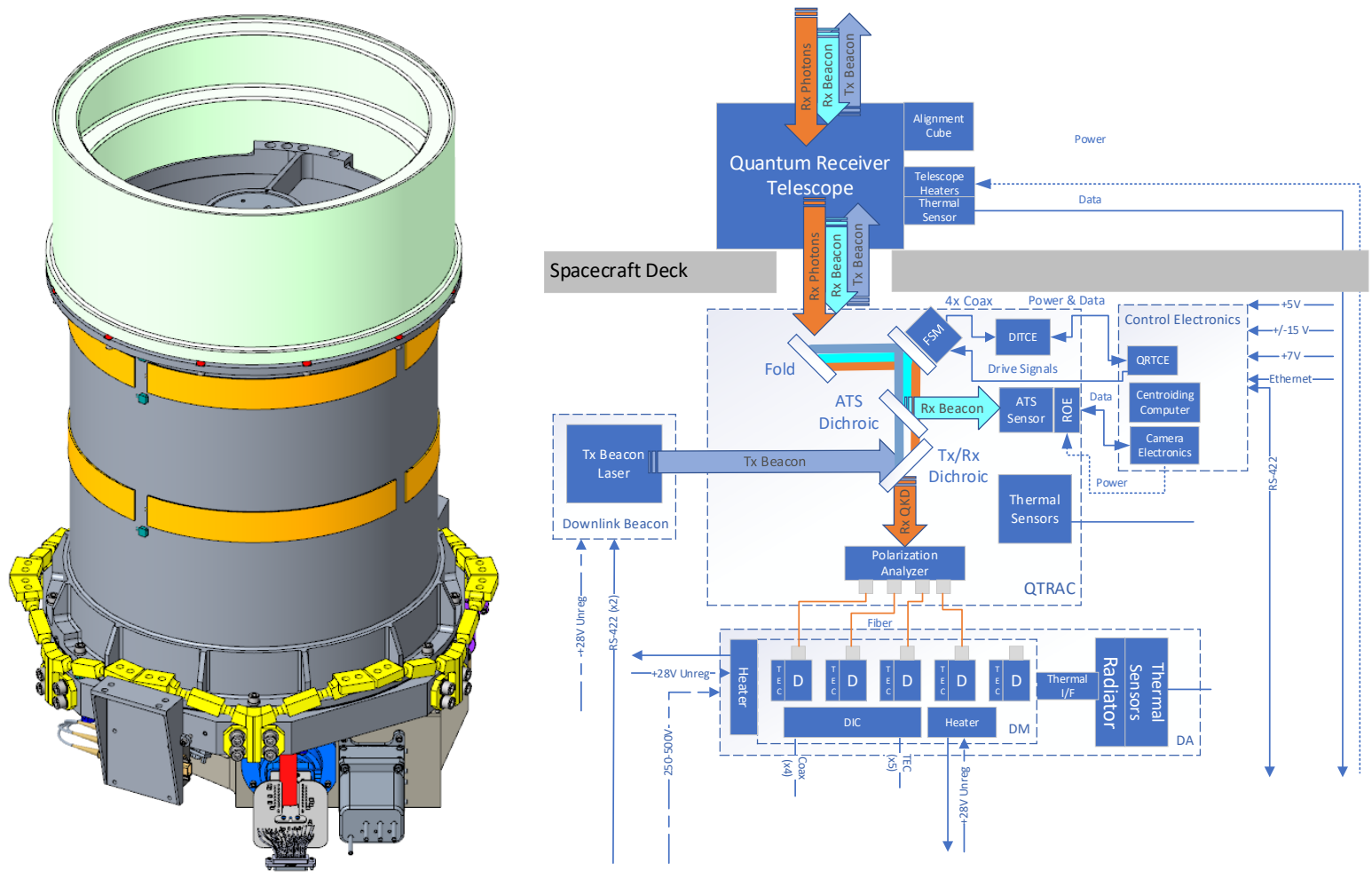

Figure 2. CAD image of QKD terminal (left) and simplified terminal architecture (right). The QKD terminal consists of a $25 \mathrm{~cm}$ frontend telescope, an optical bench and downlink sources for performing pointing and tracking operations, a polarization analyzing receiver, and a single photon counting detection system. 


\subsection{Telescope}
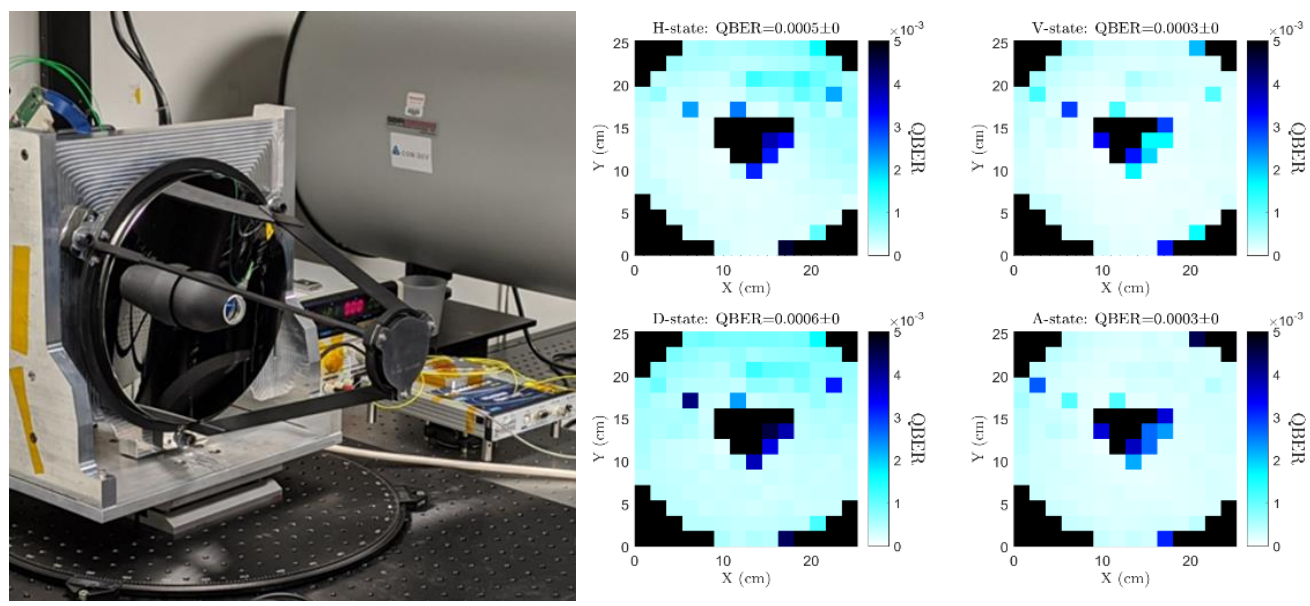

Figure 3 The breadboard QKD terminal telescope (left) is an on-axis catadioptric design with a $25 \mathrm{~cm}$ primary mirror which has been demonstrated to maintain transmitted polarization states with average quantum bit error rate below $0.03 \%$ (right).

The front-end optical receiver for the QKD payload is an afocal $25 \mathrm{~cm}$ on-axis catadioptric telescope designed to operate at multiple wavelengths in order to reformat the received uplink signals to a $17 \mathrm{~mm}$ input beam for the QTRAC. The telescope features a wide field of view (FOV) of 0.3 degrees half-angle, in order to accommodate for potential mis-pointing of the spacecraft bus towards the ground station. A breadboard version of this telescope (see Figure 3) was recently produced by Honeywell for CSA, and its Quantum Bit Error Rater (QBER) performance has been measured at the Institute for Quantum Computing [5]. Despite the requirement for a wide field of view, the on-axis catadioptric design was preferred over an allreflective three-mirror anastigmat as the on-axis design is lower mass and has inherently better performance in terms of polarization extinction ratio, which is the major driver of QBER.

The opto-mechanical design, shown in Figure 2, is based on Honeywell's aluminum diamond machining processes, with aluminum mirrors and structure. Updates from the breadboard include the replacement of the spider structure with a solid aluminum tube (for improved stray-light performance and mechanical stability), improvements to the primary mirror mounting interface to reduce mirror distortion, and the incorporation of an internal baffle near the field lens. An innovative titanium bipod flexure design (seen in yellow in Figure 2) has been implemented to provide compliance in the $\mathrm{z}$ direction (optical axis) and reduce distortions of the primary mirror from mounting to the spacecraft panel in order to meet the stringent wavefront error requirements of the system. Finally, a short baffle has been incorporated into the design in order to reduce the risk of sun-blinding during ground station acquisition, where there is a risk that the payload may be looking near the Earth's limb.

\subsection{QTRAC Assembly}

Once the uplink beacon and quantum signal beams have been received by the telescope they are passed to the pointing and tracking assembly. The tracking assembly is a modified version of Honeywell's commercially-oriented Optical Pointing and Tracking Relay Assembly for Communications (OPTRAC) which has been developed for LEO optical intersatellite links (OISL); both of these systems are shown in Figure 4. The QTRAC is based on a modified OPTRAC layout which has been adapted in order to encompass the larger field of view and larger collimated beam required for the QEYSSat mission. The de-lidded QTRAC shown in Figure 4 comprises a fold mirror, to accept the incoming beam from the telescope at nominal incidence, a fine-steering mirror (FSM), and various dichroic mirrors for separating and combining received and transmitted beacon and quantum signals.

As discussed previously, the QEYSSat mission uses the satellite attitude control system to perform coarse tracking of the ground station, hence the QKD terminal optics require a wide FOV to accommodate potential spacecraft mis-pointing. To perform acquisition and fine tracking functions over this wide FOV Honeywell is making use of a pixelated sensor, leveraging techniques developed on the CALTRAC star tracker and JWST FGS. The uplink beacon signal entering QTRAC is directed to an InGaAs imaging array, which centroids the uplink beacon at $10 \mathrm{kHz}$ and permits tracking anywhere within the FOV at an accuracy of $1 / 15^{\text {th }}$ pixel $(<0.3$ arcsec, real-space $)$. 
The use of a pixelated array confers a number of advantages over quadrant photodiode sensors which are commonly used as tracking sensors in similar applications including previous experiments to develop the QEYSSat mission concept [3]. First, background and stray light sources as well as radiation hits over the full area of the device have a reduced impact, since the pixelated sensor readout is sub-windowed to a small area of interest. Second, the pixelated sensor can provide accurate centroid measurements anywhere within the sensor array, and therefore does not need to maintain a tight alignment to the quantum uplink $(\mathrm{Rx})$ boresight so long as the boresight alignment can be re-calibrated. This can result in substantially looser alignment tolerances during assembly, as well as over the thermal operational range. Finally, the pixelated sensor offers greater tracking sensitivity, delivering a lower noise-equivalent angle than equivalent quadrant photodiodes.

Use of an InGaAs tracking sensor also allows for an NIR uplink beacon in order to maximize wavelength distance from the sensitive single-photon QKD detectors-which are silicon-based. Radiation testing up to a 10-year lifetime has been completed on the selected InGaAs sensor, showing acceptable performance at end of life. Since the QEYSSat mission has a nominal one-year duration, radiation susceptibility is not a concern. The pointing controller uses the position of the received beacon signal on the tracking sensor to drive a fine steering mirror to correct for both satellite pointing errors as well as platform jitter, thus maximizing the coupling of uplink photons into the quantum receiver.

Following the pointing and tracking system, the QKD uplink signal is directed to an optical relay, where it is re-formatted to match the $5 \mathrm{~mm}$ acceptance pupil for the polarization analyzer and narrowband spectral filter. Finally, the downlink beacon and QKD signal are passed to the QTRAC via polarization-maintaining fiber and are expanded to weakly diverging beams using fiber collimators. Note that quantum uplink and downlink will not occur simultaneously, so the QTRAC does not need to provide point ahead correction using a second mechanism. The point-ahead angle is the angular difference between the point where the beacon is seen, and the point to where the transmitter needs to aim to ensure photons hit the partner terminal. Point-ahead is required due to the motion of the satellite during the finite delay between photon transmission and reception. During QKD uplink, the uplink quantum and beacon signals originate from the same point, so point-ahead is not required at the satellite. For a quantum downlink, the point-ahead angle will be applied by tracking the uplink beacon signal to an off-center position on the pixelated ATS. In order to ensure that the ground station can always track the downlink satellite beacon, the beacon is defocused to provide a broadened beam with "passive" point-ahead.

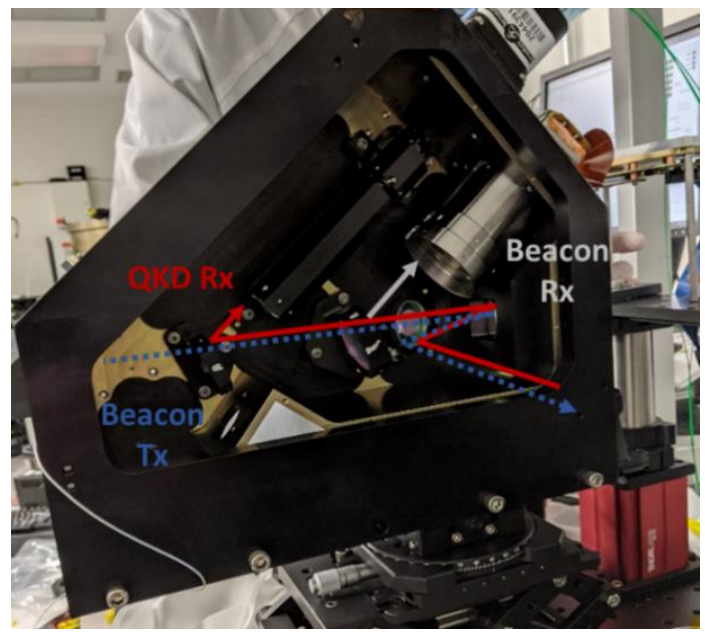

Figure 4 the QTRAC is a modified pointing and tracking relay based on the commercially-oriented OPTRAC

Finally, the received quantum signal is directed to a polarization analyzer in order to determine the initial state of the uplinked photon. The polarization analyzer uses a non-polarizing 50/50 beam-splitter in order to sort incoming photons into either the horizontal-vertical (HV) or antidiagonal-diagonal (AD) bases with equal probability. Once the incoming photons have been sorted into a particular basis (e.g. horizontal-vertical) they are then separated by polarizing beamsplitters in order to measure the polarization state. Once the uplink has been concluded, spurious measurements (e.g. wherein the uplink photon was prepared in the HV basis but detected in the AD basis) are discarded and the remaining measurements are used to generate a secure encryption key.

The alignment and optical performance of the BB QTRAC has undergone performance testing at Honeywell which has shown that the QTRAC is meeting or exceeding its requirements. To maximize performance for the quantum uplink, each optical element within the QTRAC is designed in order to maintain the fidelity of the polarized uplink signal and minimize system QBER — similar to the telescope shown in Figure 3. Under testing, the polarization extinction ratio (a major driver 
of QBER) of the QTRAC was measured to be over 450:1 in all channels, and the optical throughput of the QTRAC was measured to be $81.6 \%$.

\subsection{Detector module}

The QKD terminal uses a four-channel single-photon detection system based on silicon avalanche photodiodes (APDs) operating in Geiger mode. The silicon APDs are connected optically to the QKD terminal using multi-mode fibers, and the detectors themselves are housed on the backside of a radiative cold plate that will be used to passively cool the detectors. Previous studies have shown that APDs are highly susceptible to the space radiation environment, however the impacts of radiation damage can be mitigated by cooling the detectors in order to reduce dark counts [6,7]. The APDs in the QKD terminal will be cooled to below $-90^{\circ} \mathrm{C}$ by a combination of passive cooling from the radiator panel, and active cooling from a two-stage Peltier module integrated into the APD packages (as shown in Figure 5).
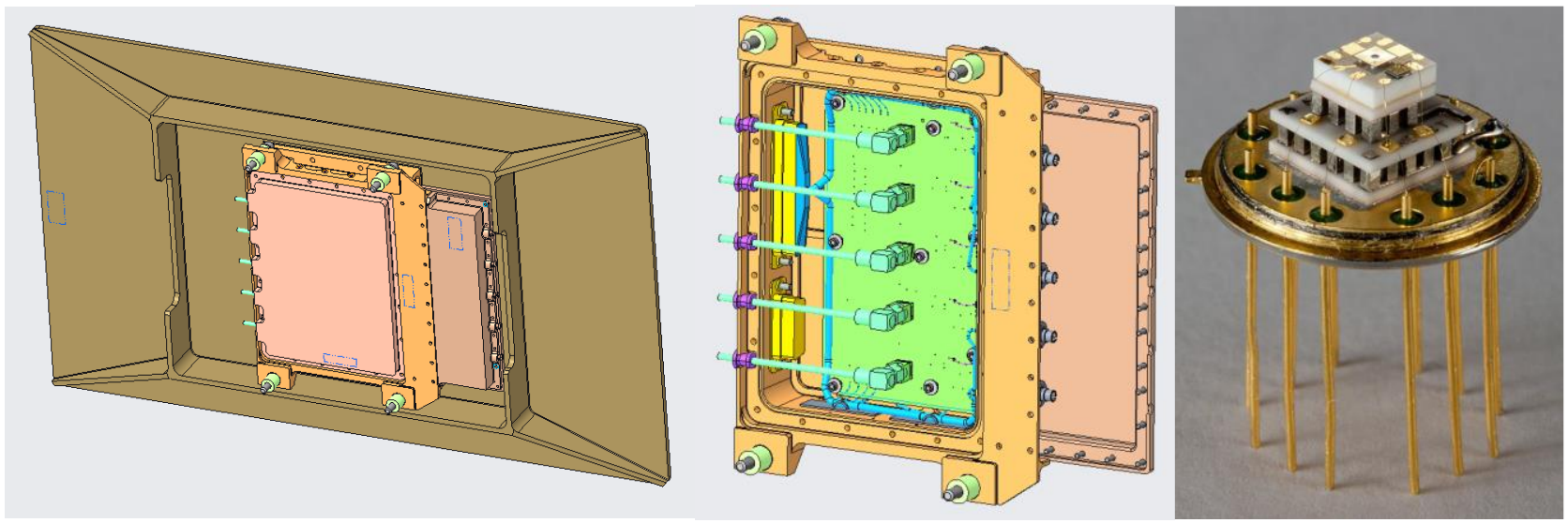

Figure 5 the detector module (left) comprises a large radiator panel for passive cooling of the detector interface card (middle) which houses the single photon counting APDs (right). The detector module is thermally isolated from the rest of the payload in order to maximize the cooling effect of the radiator.

\section{LINK ANALYSIS}

\subsection{Quantum link}

Detailed link calculations for the QKD signal uplink, and beacon uplink and downlinks have been developed to ensure that the QKD terminal is able to meet the user requirements for quantum key generation. The link budget for the quantum uplink is presented in

Table 1 . The table presents a receiver sensitivity value of $-122.7 \mathrm{dBm}$ in order to demonstrate the level of link margin for different link conditions, however in reality the receiver sensitivity for the quantum link is a dynamic value which is determined not only by the loss in the channel, but also by the amount of background light in the channel — which may vary with link conditions. The values presented in the link budget are derived from the input power level at which the instantaneous QBER falls to 5\% (the mission threshold). Integrating the instantaneous QBER, signal and decoy rates, channel loss, and background counts over the duration of a given satellite pass, and by calculating the lower bound on the secure key generation rate [8], we determine the minimum length of secure key that may be generated for a given pass. These minimum key lengths are presented for a variety of passes in Table 2.

The quantum link budget uses as its baseline a $12 \mathrm{~cm}$ diameter ground station telescope (or equivalent diverging beam), however as part of the mission goals there is interest to work with a variety of ground stations and the link budget does not preclude the use of smaller telescopes since the range loss factor due to long term beam wander (atmospheric dropout) dominates over intrinsic diffraction loss. The QEYSSat ground station is assumed to achieve diffraction-limited performance, resulting in a static transmitted wavefront error loss (Strehl) of $-0.9 \mathrm{~dB}$. The link budget assumes the use of a WCP source operating at a $400 \mathrm{MHz}$ repetition rate, as well as an entangled source operating at $100 \mathrm{MHz}$ repetition rate.

Channel loss through the atmosphere is modeled as a combination of molecular absorption, and beamspread due to longterm beam wandering and diffraction broadening. A rural atmosphere with $10 \mathrm{~km}$ visibility (a typical value near Ottawa) is used to estimate atmospheric absorption. Additionally, an accumulation of tilt errors introduced during propagation through the atmosphere results in a wandering beam in the far-field; in the long term, this effect produces greater average link loss, 
as-if the transmitter had been de-focused or had a substantially larger diffraction-limited beamspread. The beamspreading effect is calculated separately from scintillation (self-interference) effects and results in an effective beam radius of approximately $10 \mathrm{~m}$ at $600 \mathrm{~km}[9,2]$.

Table 1 Quantum uplink (Rx) budget presented at 90 degree elevation (zenith) and 48.5 degree elevation

\begin{tabular}{|c|c|c|c|}
\hline Contribution & $90^{\circ} \mathbf{E l}$ & $48.5^{\circ} \mathrm{El}$ & Justification \\
\hline Units & $(\mathrm{dB})$ & (dB) & \\
\hline Transmitter power $(\mathrm{dBm})$ & -73.0 & -73.0 & 0.5 phot / pulse @ $400 \mathrm{MHz}$ rep rate \\
\hline Gaussian aperture truncation & -0.9 & -0.9 & Assumed optimum truncation ratio at ground transmitter \\
\hline Optical Strehl & -0.9 & -0.9 & Excess WFE at transmitter re-distributes power to side-lobes \\
\hline Tx gain & 113.6 & 113.6 & Telescope gain \\
\hline Transmitter subtotal & 111.9 & 111.9 & \\
\hline Pointing error 3-sigma & -0.4 & -0.4 & $<6$ urad radial pointing accuracy \\
\hline Atmospheric absorption & -2.0 & -2.7 & $10 \mathrm{~km}$ visibility \\
\hline Atmospheric broadening & -9.0 & -10.1 & Long-term beam radius from $\mathrm{H}-\mathrm{V}$ model for beam wander \\
\hline Atmospheric scintillation & -0.3 & -1.3 & H-V model with no aperture averaging (single transmitter) \\
\hline Maximum range loss & -140.2 & -142.5 & $600 \mathrm{~km}$ orbit; $0.25 \mathrm{~m}$ on-axis receiver $\mathrm{w} / 12.5 \%$ obscuration \\
\hline Channel subtotal & -152.0 & -156.9 & \\
\hline Rx optical chain losses & -3.0 & -3.0 & Summation of optical losses from terminal optical elements \\
\hline Pol-analyzer transmission & -1.3 & -1.3 & Losses from polarization analyzer including fiber coupling \\
\hline Receiver subtotal & -4.3 & -4.3 & \\
\hline Link total & -44.4 & -49.4 & \\
\hline Rx power $(\mathrm{dBm})$ & -117.5 & -122.4 & \\
\hline Background power $(\mathrm{dBm})$ & -140.0 & -138.7 & $\begin{array}{l}39.80 \mathrm{cps}-\text { Earth blackbody, moonlight (half moon), urban } \\
\text { lights, } 15 \mathrm{~nm} \text { bandpass filter }\end{array}$ \\
\hline Detector background $(\mathrm{dBm})$ & -130.2 & -130.2 & $375 \mathrm{cps}, 350 \mathrm{ps}$ timing resolution \\
\hline Receiver sensitivity (dBm) & -122.7 & -122.7 & Power at which QBER > 5\% (pass cutoff) \\
\hline Link Margin (dB) & 5.2 & 0.3 & \\
\hline
\end{tabular}

The satellite receiver shown in Figure 2 is modeled using the combination of all reflection and transmission terms for each element in the optical chain. Wavefront error and pointing losses in the receiver are accounted for in a worst-case $80 \%$ throughput of the spatial filter (pinhole), which is achievable without the need for diffraction-limited performance. The link budget also considers background count contributions from detector dark current, as well as light contributions from reflected moonlight, and human nighttime activity. The magnitude of the background light is maintained at a low level via spatial filtering, which results in a ground receiver footprint of $\approx 22 \mathrm{~m}$ wide.

In order to ensure that the mission is compatible with a variety of quantum sources, the optical payload is designed to accept single-photon uplink signals between $780 \mathrm{~nm}$ and $795 \mathrm{~nm}$ with polarization-encoding in the horizontal, vertical, antidiagonal and diagonal bases. The downlink source also uses polarization-encoding in linear states and is designed to operate at $850 \mathrm{~nm}$.

Table 2 Quantum keys generated for overhead passes at various maximum elevation angles and for various quantum sources.

\begin{tabular}{|c|c|c|c|}
\hline $\begin{array}{c}\text { Maximum } \\
\text { elevation }\end{array}$ & $\begin{array}{c}\text { Pass } \\
\text { cutoff }\end{array}$ & $\begin{array}{c}\text { WCP key } \\
\text { (kbits) }\end{array}$ & $\begin{array}{c}\text { Entangled } \\
\text { Key (kbits) }\end{array}$ \\
\hline $\mathbf{3 3}^{\circ}$ & $\mathbf{3 0}^{\circ}$ & NA & NA \\
\hline $\mathbf{4 2}^{\circ}$ & $\mathbf{3 0}^{\circ}$ & NA & 1.6 \\
\hline $\mathbf{5 3}^{\circ}$ & $\mathbf{4 0}^{\circ}$ & NA & 18.2 \\
\hline $\mathbf{6 6}^{\circ}$ & $\mathbf{4 0}^{\circ}$ & 19.0 & 42.0 \\
\hline $\mathbf{7 1}^{\circ}$ & $\mathbf{4 0}^{\circ}$ & 27.7 & 50.9 \\
\hline $\mathbf{8 6}^{\circ}$ & $\mathbf{4 0}^{\circ}$ & 43.4 & 66.0 \\
\hline
\end{tabular}




\subsection{Beacon sensitivity}

As described previously, the QEYSSat mission requires the use of both uplink and downlink beacons in order to enable acquisition and tracking for both the transmitter and the receiver. The acquisition and tracking sensor may acquire the beacon signal with unmodulated input irradiance of $80 \mathrm{pW} / \mathrm{m}^{2}$ at the telescope entrance and is able to establish its fine-tracking loop with an input irradiance at $8 \mathrm{nW} / \mathrm{m}^{2}$. The primary QEYSSat downlink beacon is designed to deliver an unmodulated irradiance of $>10 \mathrm{nW} / \mathrm{m}^{2}$ at the ground station.

In the primary mission the satellite acquisition is initiated at a low angle of elevation, resulting in significant distortion of the beacon due to atmospheric scintillation and the increased propagation distance. These losses amount to an additional link loss of over $25 \mathrm{~dB}$ with respect to the values in

Table 1. Accordingly, the uplink beacon power and divergence are oversized with respect to the expected tilt-errors induced by the atmosphere, as well as the expected uncertainty on the spacecraft position.

\section{SECONDARY PAYLOAD}

The QEYSSat mission also incorporates as a secondary payload an optical communications terminal developed by Honeywell to address the high performance, low cost objectives for optical communication within large LEO constellations. The secondary payload will be a flight demo mission of the OISL concept which takes advantage of similarities with the primary payload to simplify the terminal. Specifically, the terminal will not have independent pointing, and will rely on the bus attitude control to track the ground stations. The mission will also demonstrate links to ground, using the same ground terminal as the primary payload, rather than intersatellite links. Although an intersatellite link will be demonstrated if a compatible partner terminal becomes available.

This payload consists of an optical head unit (OHU) and an associated controller electronics unit with an integrated transceiver. The OHU, shown in Fig 4, consists of the OISL terminal telescope and OPTRAC, with a slightly adjusted mechanical interface for compatibility with the QEYSSat bus. The telescope is a $10 \mathrm{~cm}$ aperture all aluminum three mirror anastigmat (TMA). The OPTRAC is the inspiration for the QTRAC of the primary payload, with an octal cell tracking sensor, fine steering mirror, point ahead correction steering mirror, and beamsplitters to separate transmitted and received signals. The communication terminal does not use a dedicated beacon for tracking. Instead, a small percentage of the relatively high-powered communication carrier is directed towards the tracking sensor to drive the controller.
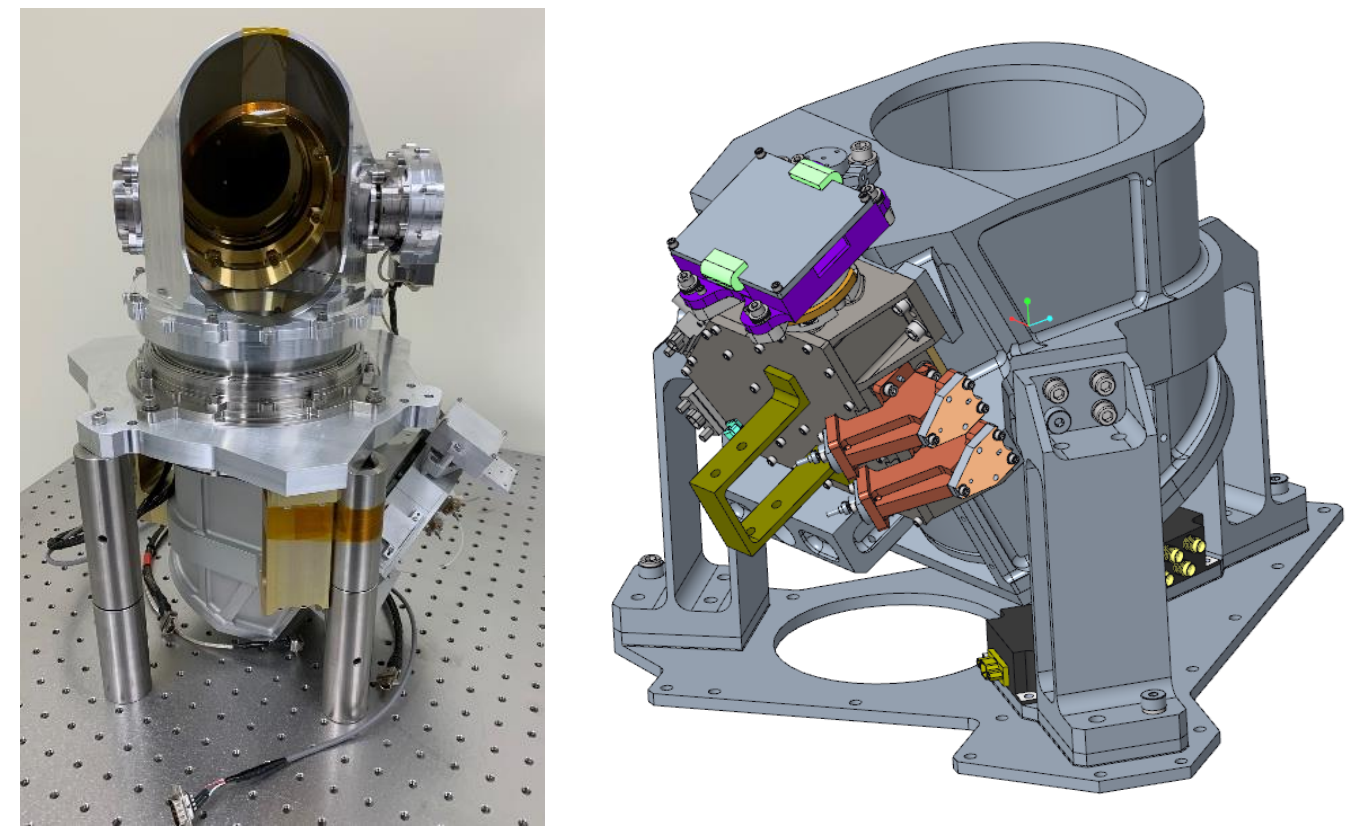

Figure 6. Honeywell's commercial OISL optical head unit (left) and adapted secondary payload version (telescope and OPTRAC only) (right)

Fiber optics coupled to the OPTRAC connect to the transceiver portion of the controller electronics unit. The transceiver provides test/diagnostic functions to characterize the link quality during this demonstration mission while the controller 
portion of the unit operates the OHU, driving the pointing correction in the OPTRAC, and provides an interface to the bus in the role of payload computer.

The optical terminal is designed to provide full duplex $10 \mathrm{Gbps}$ communication via on/off keying on a $1550 \mathrm{~nm}$ intersatellite link with the transmit and receive channels separated by wavelength. In the QEYSSat mission the terminal will be used to demonstrate a classical optical communication link to the ground and may be used to provide joint characterization of quantum and classical links through the atmosphere. In addition to its role in supporting the QEYSSat primary mission, the secondary payload will serve as a technology development and demonstration flight for the terminal as a commercial product. Further details on the design of this terminal may be found in a companion paper [8].

\section{ACKNOWLEDGEMENTS}

This work is funded under contract from the Canadian Space Agency (CSA), and is the culmination of many successful Science and Technology Development Program (STDP) studies, also funded by CSA.

\section{REFERENCES}

[1] D. Rideout et al., "Fundamental quantum optics experiments conceivable with satellites--reaching relativistic distances and velocities," Class. Quantum Grav., vol. 29, p. 224011, 2012.

[2] J. P. Bourgoin et al., "A comprehensive design and performance analysis of low Earth orbit satellite quantum communication.," New Journal of Physics, vol. 15.2, p. 023006, 2013.

[3] C. J. Pugh et al., "Airborne demonstration of a quantum key distribution receiver payload," Quantum Sci. Technol., vol. 2, p. 024009 , 2017.

[4] J. Yin, et al., "Satellite-based entanglement distribution over 1200 kilometers," Science, vol. 356, pp. 1140-1144, 2015.

[5] Y. S. Lee, et al., "Optical polarimetry using a six-axis robot arm for free-space quantum communication devices," in preparation.

[6] E. Anisimova et al., "Mitigating radiation damage of single photon detectors for space applications," EPJ Quantum Technology, vol. 4, p. 10, 2017.

[7] I. D’Souza et al., "Repeated radiation damage and thermal annealing of avalanche photodiodes," under review, https://arxiv.org/abs/2007.04902

[8] X. Ma, B. Qi, Y. Zhao and H.-K. Lo, "Practical decoy state for quantum key distribution," Phys. Rev. A, vol. 72, p. $012326,2005$.

[9] C. Erven et al., "Improving free-space quantum key distribution in the turbulent atmosphere," New J. Phys., vol. 14, p. $123018,2012$.

[10] D. Hudson et al., "Optical Communication Terminal for Low-Cost Volume Production," in 2019 IEEE International Conference on Space Optical Systems and Applications (ICSOS), in press, 2019. 